\title{
Hesperidin alleviates rat postoperative ileus through anti-inflammation and stimulation of $\mathrm{Ca}^{2+}$-dependent myosin phosphorylation
}

\author{
Yong-jian XIONG ${ }^{1, \#}$, Hong-wei $\mathrm{CHU}^{2, \#}$, Yuan $\mathrm{LIN}^{2}$, Fang $\mathrm{HAN}^{3}$, Ya-chan $\mathrm{LI}^{3}$, Ai-guo WANG ${ }^{3}$, Fu-jin WANG ${ }^{3}$, Da-peng CHEN $\mathrm{CH}^{3, *}$, \\ Jing-yu WANG ${ }^{3, *}$ \\ ${ }^{1}$ Central Laboratory, the First Affiliated Hospital, Dalian Medical University, Dalian 116011, China; ${ }^{2}$ Dalian Medical University, Dalian \\ 116044, China; ${ }^{3}$ Laboratory Animal Center, Dalian Medical University, Dalian 116044, China
}

Aim: Postoperative ileus (POI) is a postoperative dysmotility disorder of gastrointestinal tract, which remains one of the most perplexing problems in medicine. In the present study we investigated the effects of hesperidin, a major flavonoid in sweet oranges and lemons, on $\mathrm{POI}$ in rats.

Methods: SD rats were administered hesperidin $\left(5,20\right.$, and $80 \mathrm{mg} \cdot \mathrm{kg}^{-1} \cdot \mathrm{d}^{-1}$, ig) for 3 consecutive days. POI operation (gently manipulating the cecum for $1 \mathrm{~min}$ ) was performed on $\mathrm{d} 2$. The gastrointestinal motility and isolated intestinal contraction were examined $1 \mathrm{~d}$ after the operation. Then the myosin phosphorylation and inflammatory responses in cecum tissue were assessed. Smooth muscle cells were isolated from rat small intestine for in vitro experiments.

Results: The gastric emptying and intestinal transit were significantly decreased in POI rats, which were reversed by administration of hesperidin. In ileum and cecum preparations of POI rats in vitro, hesperidin (2.5-160 $\mu \mathrm{mol} / \mathrm{L})$ dose-dependently increased the spontaneous contraction amplitudes without affecting the contractile frequency, which was blocked by the myosin light chain kinase (MLCK) inhibitor ML-7 or verapamil, but not by TTX. Furthermore, administration of hesperidin increased the phosphorylation of MLC $_{20}$ in the cecum tissue of POI rats. Moreover, administration of hesperidin reversed the increased levels of inflammatory cytokines, iNOS and COX-2 in cecum tissue of POI rats. In freshly isolated intestinal smooth muscle cells, hesperidin (5-80 $\mu \mathrm{mol} / \mathrm{L})$ dose-dependently increased the intracellular $\mathrm{Ca}^{2+}$ concentration as well as the phosphorylation of $\mathrm{MLC}_{20}$, which was abrogated by ML-7 or siRNA that knocked down MLCK.

Conclusion: Oral administration of hesperidin effectively alleviates rat POI through inhibition of inflammatory responses and stimulation of $\mathrm{Ca}^{2+}$-dependent MLC phosphorylation.

Keywords: hesperidin; postoperative ileus; cecum; MLCK; myosin; $\mathrm{Ca}^{2+}$; inflammation; ML-7; verapamil

Acta Pharmacologica Sinica (2016) 37: 1091-1100; doi: 10.1038/aps.2016.56; published online 27 Jun 2016

\section{Introduction}

The rat postoperative ileus (POI) refers to delayed transit of gastrointestinal content, intolerance of food, and gas retention, which often occurs in patients with acute pancreatitis or cancer who have had abdominal surgery ${ }^{[1,2]}$. As one of the postoperative dysmotility disorders of the gastrointestinal tract, postoperative ileus remains one of the most perplexing problems in medicine ${ }^{[3,4]}$. Because prolonged POI is an iatrogenic condition leading to prolonged hospitalization resulting in

\footnotetext{
\#These authors contributed equally to this work.

* To whom correspondence should be addressed.

E-mail cdp.9527@163.com (Da-peng CHEN); wangjingyus@163.com (Jing-yu WANG)

Received 2016-01-17 Accepted 2016-03-27
}

increased health care costs, the development of effective management strategies to prevent POI is clinically important ${ }^{[5]}$.

Both the decreased gastrointestinal motility and intestinal inflammatory response to bowel manipulation and surgical trauma are the main causes of $\mathrm{POI}^{[6]}$. In this study, we screened a natural drug library to identify novel drugs that could decrease inflammatory activity and stimulate gastrointestinal motility. As a result of this screening, hesperidin (Figure 1A) was shown to exert significant stimulating effects on isolated intestinal contraction. Hesperidin is the major flavonoid in sweet oranges and lemons ${ }^{[7]}$ and is abundant in certain regularly consumed foods (eg, vegetables and fruits) and some beverages $(e g \text {, tea and red wine })^{[8-10]}$. Hesperidin, like other flavonoids, has been reported to possess a wide range of biological activities, including anti-inflammatory, anti-oxidant, 
anti-allergic, hepatoprotective, anti-thrombotic, anti-viral, cardiovascular, anti-carcinogenic effects, anti-depressionlike effects and prevention of bone loss ${ }^{[4,7,10-17]}$. However, the effects of hesperidin on POI are rarely reported.

The present study was designed to characterize hesperidinexerted regulatory effects on the gastrointestinal tract and to test whether hesperidin could ameliorate POI in a rat model. POI is caused by the interplay of multiple factors, and both decreased gastrointestinal motility and intestinal inflammatory responses to bowel manipulation and surgical trauma are involved $^{[6]}$. In the case of gastrointestinal motility, the gastrointestinal motility and contraction is determined by inputs from enteric motor neurons that innervate smooth muscle cells ${ }^{[18-20]}$. The extent of myosin phosphorylation is positively correlated with smooth muscle contraction, and many smooth muscles contract via this pathway ${ }^{[21]}$. Excitation-contraction coupling occurs by $\mathrm{Ca}^{2+}$ entry via ion channels in the plasma membrane, leading to a rise in intracellular $\mathrm{Ca}^{2+} . \mathrm{Ca}^{2+}$ binding to calmodulin activates myosin light chain kinase (MLCK); subsequent phosphorylation of myosin initiates cross-bridge cycling and finally leads to smooth muscle contraction. In vivo, the effects of hesperidin on gastric emptying and intestine transit were studied to test the effects of hesperidin on gastrointestinal motility; both the extent of myosin phosphorylation and the MLCK expression in sham-operated rat intestine, POI rat intestine, and hesperidin-treated rat intestine were measured. Given that the intestinal segment is able to fulfill pivotal functions even when isolated from the body ${ }^{[22,23]}$, isolated intestinal segments were used to investigate the effects of hesperidin on gastrointestinal contraction in vitro. Moreover, the direct effects of hesperidin on MLCK-dependent myosin phosphorylation were also studied using purified MLCK and myosin, which were isolated from fresh chicken gizzards. In case of inflammation, myeloperoxidase (MPO) activity and levels of pro-inflammatory cytokines, such as nitric oxide (NO) and prostaglandin E2 (PGE-2) were evaluated to test the hesperidin induced anti-inflammatory effects on POI rats. The effects of hesperidin on inducible nitric oxide synthase (iNOS) and cyclooxygenase-2 (COX-2) expression were also studied using Western blotting.

\section{Materials and methods}

\section{Animals}

Sixty Sprague-Dawley (SD) male rats (5-7 weeks of age, weighing 140-160 g) were obtained from the Laboratory Animal Center, Dalian Medical University (Certificate of Conformity: № SYXK (Liao) 2013-0006). The experimental protocol was approved by Dalian Medical University Animal Care and Ethics Committee, and all animals used were maintained in accordance with National Institutes of Health Guide for Care and Use of Laboratory Animals (Publication № 85-23, revised 1985). The animal protocol was designed to minimize pain and discomfort to the animals. The animals were acclimatized to laboratory conditions $\left(23^{\circ} \mathrm{C}, 12 \mathrm{~h} / 12 \mathrm{~h}\right.$ light/dark, $50 \%$ humidity, ad libitum access to food and water) for two weeks prior to experimentation. Intragastric gavage administration was carried out with conscious animals, using straight gavage needles appropriate for the animal size (15-17 g body weight: 22 gauge, 1 inch length, $1.25 \mathrm{~mm}$ ball diameter). All animals were euthanized by barbiturate overdose (intravenous injection, $150 \mathrm{mg} / \mathrm{kg}$ pentobarbital sodium) for tissue collection.

\section{Reagents}

Hesperidin (purify speciation: $298 \%$ ) was obtained from Xian Yiyang Bio-TECH Co, Ltd, Xi-an, China. Hesperidin was dissolved using dimethyl sulfoxide (DMSO). TTX was purchased from Aladdin Chemistry Co Ltd (Shanghai, China). Unless otherwise indicated, chemicals were obtained from SigmaAldrich (St Louis, MO, USA).

\section{Experimental design}

Rats were housed 1 per cage, and all animals lived in a temperature-controlled room with a 12-h light-dark cycle. Food and water were available ad libitum. Animals were deprived of food for $12 \mathrm{~h}$ before the experiments. Rats were randomly divided into 5 groups, each consisting of 12 rats. Group I served as sham-operated control, and the rats underwent midline abdominal incision but without any intestinal manipulation. Group II served as the POI group. Groups III, $\mathrm{IV}$, and $\mathrm{V}$ received a treatment of 5,20 , and $80 \mathrm{mg} / \mathrm{kg}$ hesperidin, respectively, after POI induction. The rat POI model was induced according to previously described methods ${ }^{[23,24]}$. Briefly, the rats were anaesthetized with a barbiturate overdose (intravenous injection, $150 \mathrm{mg} / \mathrm{kg}$ pentobarbital sodium) and submitted to laparotomy. The rat cecum was gently manipulated (patted between hands for $60 \mathrm{~s}$ in saline-soaked gauze). Abdominal muscles and skin were then closed, and the animals were allowed to recover. Hesperidin was gavaged daily at 8:00 for 3 consecutive days, and the operation for POI was performed at 15:00 on the second day. The gastric emptying, intestine transit, isolated intestinal contraction and other studies were performed $1 \mathrm{~d}$ after the operation.

\section{Gastric emptying and intestinal transit studies}

Gastric emptying and intestinal transit was measured according to previously described methods with some modifications ${ }^{[24]}$. Briefly, $2 \mathrm{~mL}$ of a distilled water solution containing 1.5\% methylcellulose and anthracotic marker (MAM) was given intragastrically, and the rats were killed by $\mathrm{CO}_{2}$ inhalation after $30 \mathrm{~min}$. Gastric emptying was calculated as [(gavaged MAM - left MAM in gastric)/gavaged MAM], and intestine transit was calculated as (the length between where the marker achieved in intestine and pylorus/the length between the pylorus and anus).

\section{Tissue preparation and intestinal contractility determination}

Small intestinal preparations were prepared according to the methods described previously with slight modification ${ }^{[25-27]}$. Intestinal preparations were cut into approximately $2 \mathrm{~cm}$ in length and then allowed to equilibrate in aerated Krebs buffer for $60 \mathrm{~min}$. The resting tension was set at approximately $1.0 \mathrm{~g}$. Contractile amplitude (contractility) and contractile frequency 
of the intestinal preparations were recorded by BL-420F Biological System (Chengdu TME Technology Co, Ltd, China). The contractility and frequency were recorded at an identical time-interval for each assay with the same start and stop time to compare the amplitude before and after drug treatment in different assay conditions. The mean amplitude and frequency were calculated from 6 independent assays.

\section{ELISA assays}

The levels of cecum MPO, cecum pro-inflammatory cytokines, including tumor necrosis factor-alpha (TNF-a), interleukin (IL)-1 $\beta$, and IL-6, were determined using an ELISA kit (R\&D Systems, Minneapolis, MN, USA) according to the manufacturer's instructions. The levels of the cecum pro-inflammatory mediators NO and PGE-2 were assayed by the Griess reaction method using a commercial kit (R\&D Systems, USA).

\section{Protein purification and myosin phosphorylation/ $\mathrm{Mg}^{2+}$-ATPase activity determination}

The myosin and MLCK used in the assay were purified from fresh chicken gizzard smooth muscle using methods described previously ${ }^{[28,29]}$. The procedures for determining the $\mathrm{Ca}^{2+}-$ dependent phosphorylation of myosin and $\mathrm{Mg}^{2+}$-ATPase activities of smooth muscle myosin were conducted according to previously described methods ${ }^{[29,30]}$. Briefly, $\mathrm{MLC}_{20}$ phosphorylation was carried out in $20 \mathrm{mmol} / \mathrm{L}$ Tris- $\mathrm{HCl}$ (pH 7.4) buffer containing $1 \mathrm{mmol} / \mathrm{L}$ dithiothreitol (DTT), $5 \mathrm{mmol} / \mathrm{L} \mathrm{MgCl}_{2}$, $60 \mathrm{mmol} / \mathrm{L} \mathrm{KCl}, 2 \mathrm{mmol} / \mathrm{L}$ ethylene glycol bis ( $\beta$-aminoethyl ether), $N, N^{\prime}$-tetraacetic acid (EGTA), $4 \mu \mathrm{mol} / \mathrm{L}$ myosin and $2 \mathrm{mmol} / \mathrm{L}$ ATP. The densitometry extent of phosphorylation of $20-\mathrm{kDa}$ regulatory light chain of myosin $\left(\mathrm{MLC}_{20}\right)$ was measured by Scion Image software (Scion Co Ltd, USA). The extent of phosphorylation of $\mathrm{MLC}_{20}$ was a relative value calculated from phosphorylated $\mathrm{MLC}_{20} /$ total $\mathrm{MLC}_{20}$.

\section{Western blot analysis}

The phosphorylation of $\mathrm{MLC}_{20}$ in cecum isolated from POI rats was examined by Western blot analysis as described previously $^{[31]}$. Cecum segments were isolated from the rats and were then immediately stored in liquid nitrogen. Total protein was isolated from the full thickness of intestinal wall using a Total Protein Extraction Kit (KeyGEN Bio TECH, China). The blots on nitrocellulose filter membrane were probed with a phosphor-myosin light chain 2 (Ser 19) antibody (1:1000) (№ 3671, Cell Signaling Technology, Inc USA), a myosin light chain 2 (total myosin light chain) antibody (1:1000) (\#3672, CST, USA) or a smooth muscle MLCK antibody $(1: 1000)$ (Abcam Ltd UK) at $4^{\circ} \mathrm{C}$ with gentle shaking, overnight. The bands were detected and quantified using a MultiSpectral imaging system (UVP, Cambridge, UK).

\section{Measurements of $\left[\mathrm{Ca}^{2+}\right]_{i}$ in smooth muscle cells}

Smooth muscle cells were isolated from the rat small intestine as previously described with slight modifications ${ }^{[32,33]}$. Briefly, intestinal segments were digested for $30 \mathrm{~min}$ at $31^{\circ} \mathrm{C}$ in HEPES medium containing $0.1 \%$ type II collagenase and $0.01 \%$ trypsin inhibitor. The cells were filtered through a $500 \mathrm{~mm}$ Nitex filter and centrifuged at 1000 rounds per minute for $10 \mathrm{~min}$. The cells were from both the longitudinal and the circular intestinal smooth muscle layers. Pre-experiment evaluation showed no significant differences in the pharmacology of isolated longitudinal smooth muscle cells and circular smooth muscle cells. The changes in intracellular free $\mathrm{Ca}^{2+}$ concentration $\left(\left[\mathrm{Ca}^{2+}\right]_{\mathrm{i}}\right)$ was measured by fluorescence measurement using $\mathrm{Ca}^{2+}$ indicator Fluo-3 AM (Dojindo, Japan) as described previously $^{[34]}$ and by a laser scanning confocal microscope (LSCM) (Radiance 2000; Bio-rad, Hertfordshire, UK). The intestinal smooth muscle cells were loaded with Fluo-3 AM (5 $\mu \mathrm{mol} / \mathrm{L}$, $45 \mathrm{~min})$.

\section{Cell transfection}

Caco-2 cells were transfected with Lipofectamine 2000 (Invitrogen) and MLCK-targeted or a control small interfering RNA (siRNA) oligos (Dharmacon, Lafayette, Co, USA) according to the manufacturer's instructions (Takara Biotechnology (Dalian) Co, Ltd). The efficiency of gene silencing was confirmed by Western blotting.

\section{Statistical analysis}

A one-way ANOVA was used where three or more groups of data were compared. Student $t$ tests were used to compare two groups of data. The data are expressed as the mean \pm SD. The data followed a normal distribution and each group had equal variances. To further evaluate the data, a KruskalWallis rank sum test was also used. All experiments were repeated at least three times, and a $P$ value of less than 0.05 was considered statistically significant.

\section{Results \\ Effects of hesperidin on gastric emptying and intestinal transit in POI rats}

The cytotoxicity of hesperidin was studied using primary cultured intestinal smooth muscle cells and hesperidin; the dose range of 5-1280 $\mu \mathrm{mol} / \mathrm{L}$ showed almost no cytotoxicity effects (data not shown). Pre-experiments were performed for selecting the dose range of hesperidin. Based on these pre-experiments and preliminary results reported by other researchers, 5-80 mg/ kg hesperidin was selected for animal experiments, and 5-80 $\mu \mathrm{mol} / \mathrm{L}$ hesperidin was selected for cell and tissue experiments $^{[35,36]}$.

Gastric emptying and intestinal transit in hesperidin-treated sham-operated rats were faster than those in rats not receiving treatment, especially in the dose group above $20 \mathrm{mg} / \mathrm{kg}$ $(P<0.01, n=10$ rats) (Figure $1 \mathrm{~B})$. The gastric emptying and intestinal transit in POI rats were slower than those in sham rats $(P<0.01, n=10$ rats). The gastric emptying and intestinal transit in hesperidin-treated $(5,20$, and $80 \mathrm{mg} / \mathrm{kg})$ POI rats were faster than those in POI rats $(P<0.01, n=10$ rats) (Figure $1 C)$. The results above indicated that hesperidin significantly reversed the decreased gastrointestinal motility in POI rats. 
A<smiles>COc1cc(O)c2c(c1)OC(c1ccc(OC)c(O)c1)CC2O</smiles>
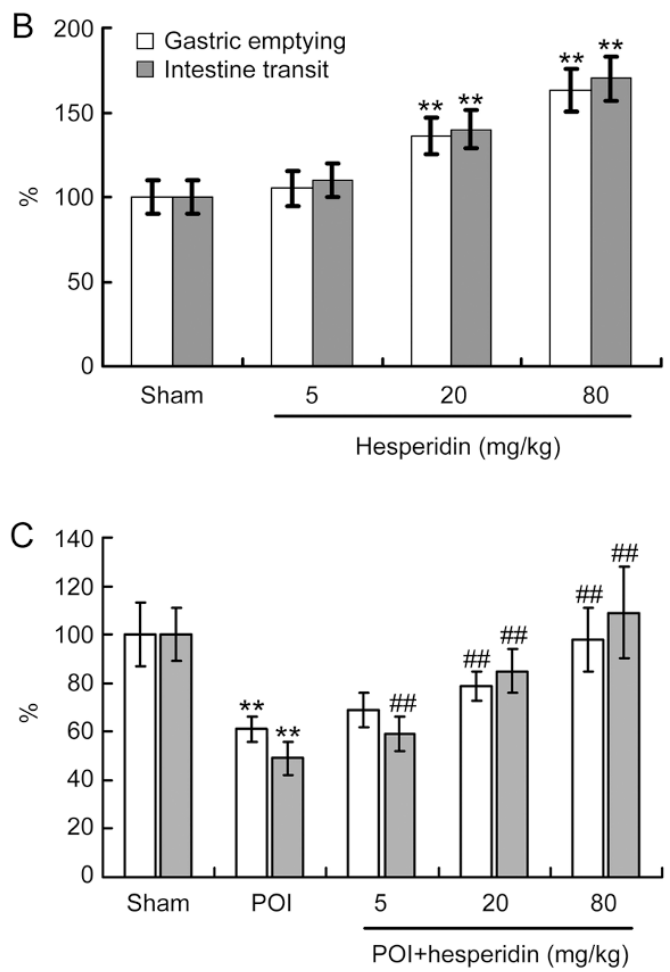

Figure 1. Effects of hesperidin on gastrointestinal motility. (A) Chemical structure of hesperidin. Effects of hesperidin on gastric emptying and intestinal transit in (B) sham-operated rats and in (C) postoperative ileus (POI) rats. Gastric emptying was calculated as (gavaged methylcellulose and anthracotic marker (MAM - left MAM in gastric)/gavaged MAM) and intestine transit was calculated as (the length between where the marker achieved in intestine and pylorus/the length between pylorus to anus). Data in sham group are set to a relative value of $100 \%$ and expressed as the mean $\pm S D$. Other data are the relative values compared with sham. ${ }^{* *} P<0.01$ compared with the sham. ${ }^{\# \#} P<0.01$ compared with $\mathrm{POI}$ group. $n=10$ rats.

\section{Effects of hesperidin on the contraction of bowel preparations}

As shown in Figure 2, the contractility of ileal preparation (3 $\mathrm{cm}$ proximal to cecum) isolated from POI rats was increased by incubation with hesperidin in a concentration range of 2.5-160 $\mu \mathrm{mol} / \mathrm{L}$ with an $\mathrm{ED}_{50}$ of $40 \mu \mathrm{mol} / \mathrm{L}$, but the contractile frequency was not significantly affected $(P<0.01, n=6$ rats) (Figure 2A). Hesperidin-induced intestinal contraction was not significantly affected by TTX (a potent neurotoxin which blocks action potentials in nerves), suggesting that the hesperidin-induced intestinal contraction was mainly mediated by a direct effect on smooth muscle. The stimulatory effect of hesperidin was significantly blocked by verapamil $(0.1 \mu \mathrm{mol} / \mathrm{L}$,
L-type $\mathrm{Ca}^{2+}$ channel blocker) and ML-7 $(5 \mu \mathrm{mol} / \mathrm{L}, \mathrm{MLCK}$ inhibitor), which indicated that $\mathrm{Ca}^{2+}$-dependent activation of MLCK is involved in hesperidin-induced stimulation of intestinal contraction (Figure 2B). Hesperidin also significantly stimulated the contraction of cecum preparations. The hesperidin-induced cecum contractions were also not affected by TTX but were significantly blocked by verapamil and ML-7 (Figure 2C).

\section{Effects of hesperidin on myosin phosphorylation}

Cecum segments were isolated from sham-operated rats, POI rats and $\mathrm{POI}+$ hesperidin rats and immediately stored in liquid nitrogen. Total protein was isolated from the full thickness of cecum preparations. The phosphorylation of $\mathrm{MLC}_{20}$ in the cecum was examined by Western blot analysis. As shown in Figure 3, the phosphorylation of $\mathrm{MLC}_{20}$ but not MLCK in the cecum was decreased in the POI group $(P<0.01, n=4$ rats $)$. The changes in phosphorylation of $\mathrm{MLC}_{20}$ could be reversed by hesperidin.

\section{Potential target of hesperidin-induced intestinal contraction}

The phosphorylation of $\mathrm{MLC}_{20}$ in the cecum preparation was increased by incubation with hesperidin $(P<0.01, n=4$ experiments). To find the target through which hesperidin enhanced the phosphorylation of $\mathrm{MLC}_{20}$, the direct effects of hesperidin on myosin phosphorylation in buffer mainly that contained purified myosin and MLCK was carried out.

As shown in Figure 4, phosphorylation of $\mathrm{MLC}_{20}$ was increased by incubation with hesperidin in the concentration range of $5-80 \mu \mathrm{mol} / \mathrm{L}(P<0.01, n=4$ experiments). The total myosin and MLCK expression was not significantly affected by hesperidin. The $\mathrm{Mg}^{2+}$-ATP activity was also increased by hesperidin $(P<0.01, n=4$ experiments).

To test the role of $\mathrm{Ca}^{2+}$ in hesperidin induced intestinal contraction, $\left[\mathrm{Ca}^{2+}\right]_{\mathrm{i}}$ was studied. After the intestinal smooth muscle cells were incubated with 5, 20, and $80 \mu \mathrm{mol} / \mathrm{L}$ hesperidin for $5 \mathrm{~min},\left[\mathrm{Ca}^{2+}\right]_{\mathrm{i}}$ was increased $(P<0.01, n=4$ experiments) (Figure 5).

In the presence of ML-7, the hesperidin-induced phosphorylation of $\mathrm{MLC}_{20}$ in intestinal smooth muscle cells was significantly blocked; after siRNA knockdown of MLCK, the hesperidin-induced phosphorylation of $\mathrm{MLC}_{20}$ in intestinal smooth muscle cells was almost abolished, which further indicated that hesperidin increased gastrointestinal through activating MLCK, leading to the myosin phosphorylation (Figure 6).

\section{Effects of hesperidin on inflammatory responses in POI rats}

The inflammatory responses in the ileum and the cecum were studied in POI rats. The MPO activity, levels of proinflammatory cytokines (TNF- $\alpha$, IL-1 $\beta$, and IL-6), and inflammatory mediators (NO and PGE-2) in the cecum isolated from POI rats were increased compared to those in the sham group $(P<0.01, n=6$ rats $)$. At the dose range of 5 to $80 \mathrm{mg} / \mathrm{kg}$, hesperidin reversed the changes in MPO activity and cytokine and inflammatory mediator levels ( $P<0.01, n=6$ rats) (Figure $7 \mathrm{~A})$. The high expression of iNOS and COX-2 in cecum tissue iso- 
A
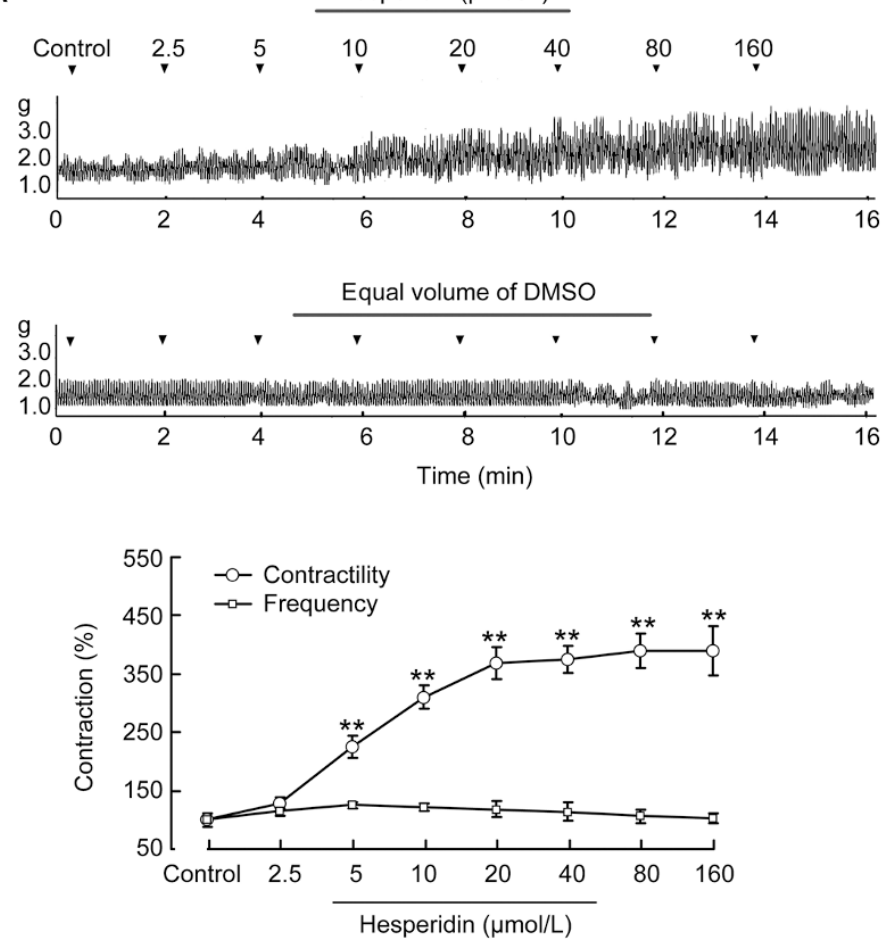

C

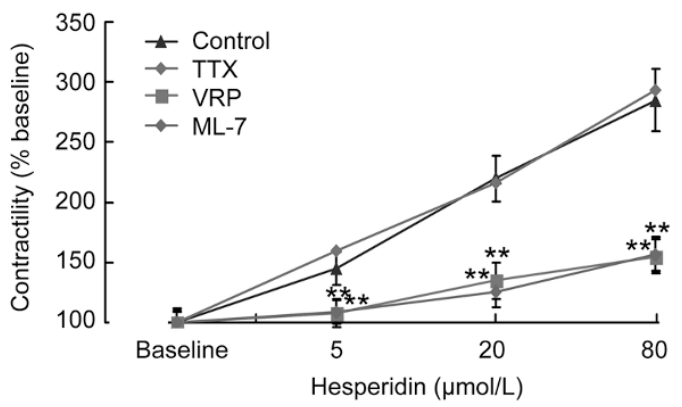

B
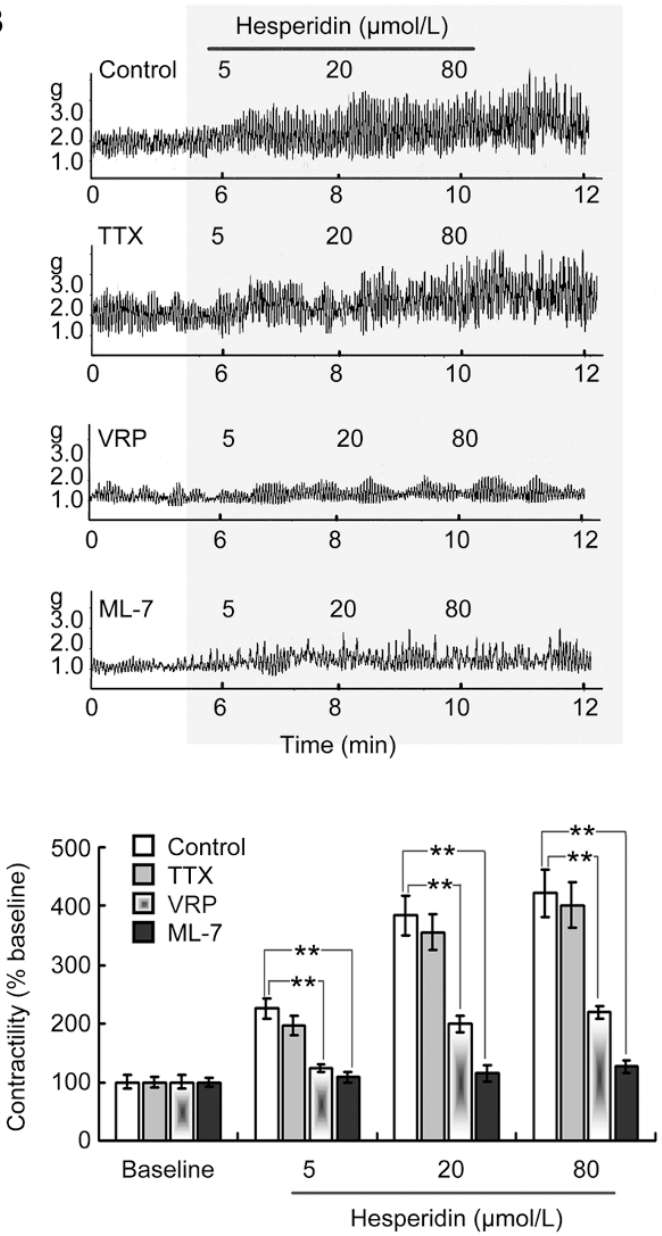

Figure 2. Hesperidin induced contraction in ileum and cecum preparations isolated from postoperative ileus (POI) rats. Ileum segment $3 \mathrm{~cm}$ proximal to the cecum was used in this study. (A) Representative recordings showing the effects of hesperidin on ileum contractility. In the lower plot, data are expressed as mean \pm SD. Data in POI group is set to $100 \%$ (control), and other data are the relative value compared with control. (B) Effects of hesperidin on ileum contraction in the presence of different blockers. Data obtained from POI rat before hesperidin treatment is set to a relative value of $100 \%$ (baseline). Other data are the relative values compared with baseline. (C) Effects of hesperidin on the contraction of cecum preparation. Data obtained from $\mathrm{POI}$ rat before hesperidin treatment is set to a relative value of $100 \%$ (baseline). All the data are expressed as the mean $\pm \mathrm{SD}$. ${ }^{* *} \mathrm{P}<0.01$ compared with baseline or control, $n=6$ tissues.

lated from POI rats were also reversed by hesperidin $(P<0.01$, $n=6$ rats) (Figure 7B).

The MPO activity and levels of pro-inflammatory cytokines and inflammatory mediators in ileum tissue $(3 \mathrm{~cm}$ proximal to the cecum) were also elevated compared with those in the sham group ( $P<0.01, n=6$ rats). All of these changes could be reversed by hesperidin $(P<0.01, n=6$ rats) (Figure 8$)$. There were no inflammatory responses in ileum tissue $(10 \mathrm{~cm}$ proximal to the cecum), and inflammatory responses in the colon were also not found.

\section{Discussion}

In summary, both the inflammatory responses and decreased gastrointestinal motility in POI rats were ameliorated by gavage administration of hesperidin.

The decreased gastrointestinal motility, including slow gastric emptying and intestine transit in POI rats, was significantly reversed by gavage administration of hesperidin. Contraction of intestinal preparations isolated from POI rats was also stimulated by hesperidin. Hesperidin-induced intestinal contraction was blocked by verapamil but not by TTX, sug- 

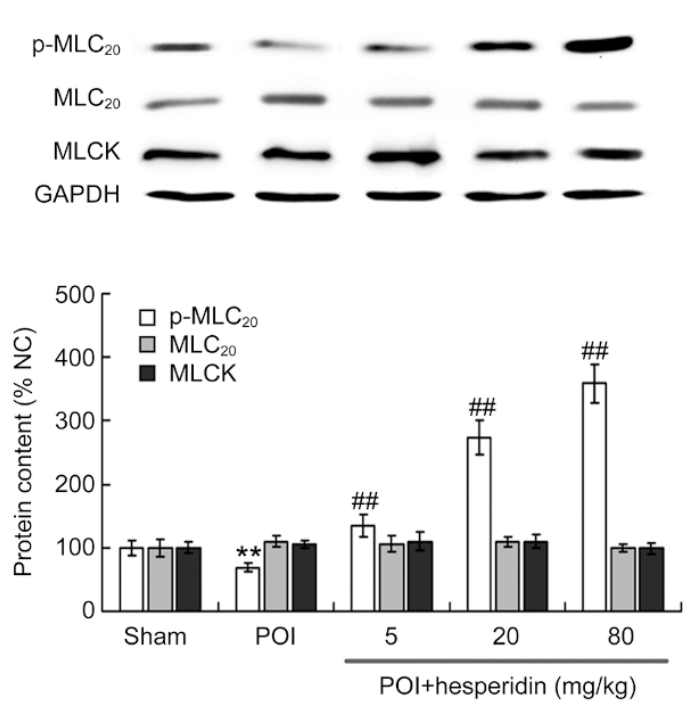

Figure 3. Effects of hesperidin on myosin phosphorylation in cecum preparations isolated from postoperative ileus (POI) rats. Data obtained in sham-operated rats are set to a relative value of $100 \%$ and expressed as the mean \pm SD. ${ }^{* *} P<0.01$ compared with the sham; ${ }^{\# \#} P<0.01$ compared with $\mathrm{POI}$ group, $n=4$ rats. $\mathrm{MLC}_{20}, 20-\mathrm{kDa}$ regulatory light chain of myosin; MLCK, myosin light chain kinase.

gesting that hesperidin induced intestinal contraction is $\mathrm{Ca}^{2+}-$ dependent and that no neural control is involved. Targeted deletion of MLCK in gastrointestinal smooth muscle resulted in severe gut dysmotility characterized by weak peristalsis and dilation of the digestive tract, suggesting that MLCK is central to smooth muscle contraction and required for gastrointestinal motility ${ }^{[37]}$. Hesperidin significantly increased the extent of myosin phosphorylation as well as $\mathrm{Mg}^{2+}$-ATP activity in vitro.

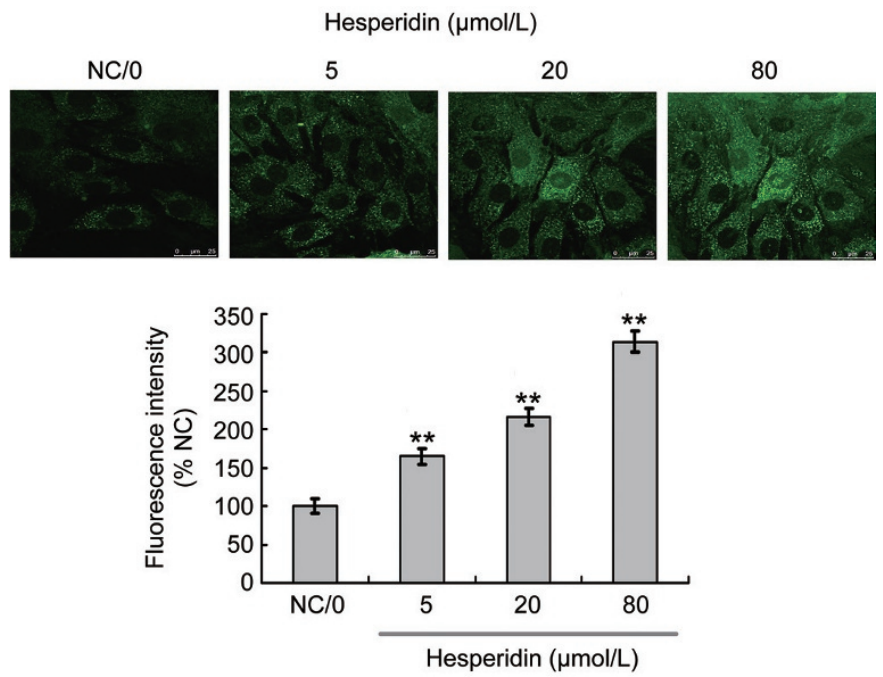

Figure 5. Effects of hesperidin on cellular $\mathrm{Ca}^{2+}$ influx $\left(\left[\mathrm{Ca}^{2+}\right]_{\mathrm{i}}\right)$ in smooth muscle cells. Data in normal control group (no hesperidin treated) are set to a relative value of $100 \%$ (normal control, NC) and expressed as the mean \pm SD. ${ }^{* *} P<0.01$ compared with the NC. $n=4$ experiments.

The extent of myosin phosphorylation is positively related to smooth muscle contraction ${ }^{[21]}$. In the intestinal smooth muscle cells with si-RNA-knock down of MLCK, the hesperidin induced stimulatory effect was almost abolished, indicating the key role of MLCK in hesperidin induced intestinal contraction.

The cause of POI is not completely understood, and its treatment remains limited. Recent reports indicate the presence of inflammatory changes in the mucosa and the enteric nervous system of patients with functional bowel disorder ${ }^{[9]}$. Abdomi-
A
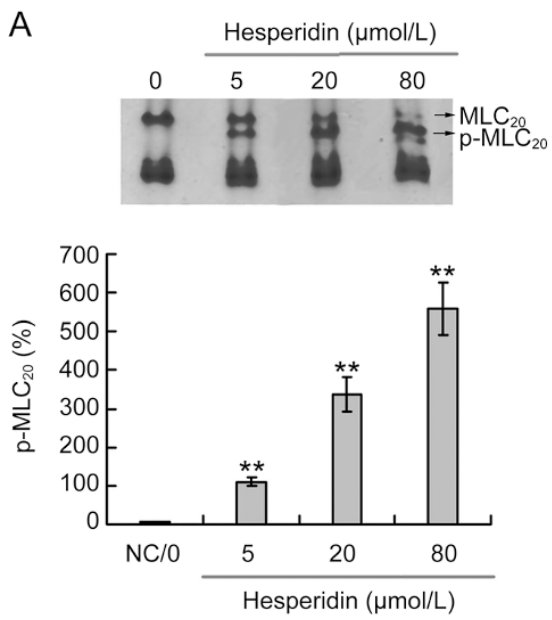

B
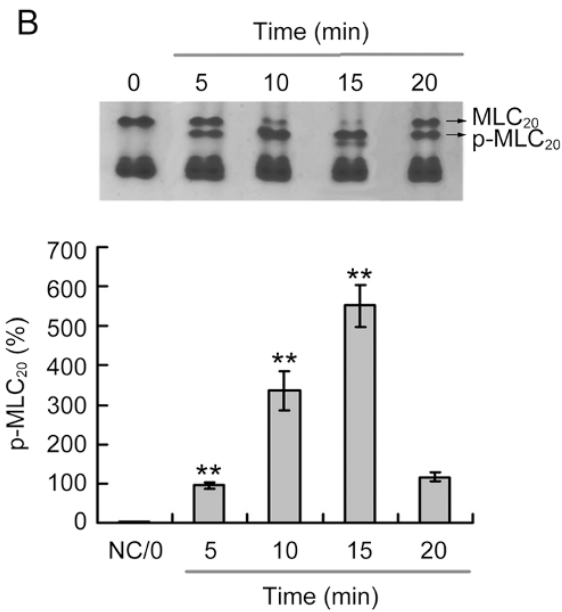

C

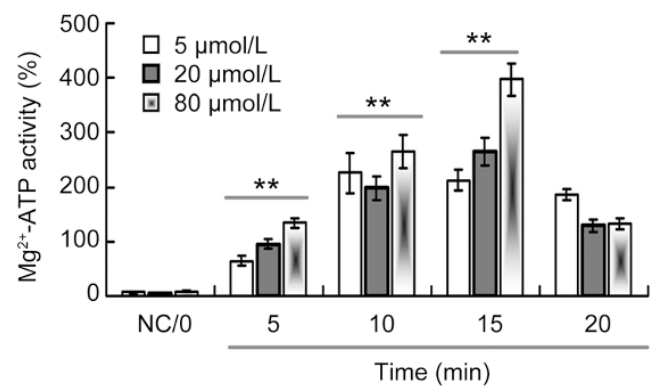

Figure 4. Effects of hesperidin on the phosphorylation of 20-kDa regulatory light chain of myosin ( $\left.\mathrm{p}-\mathrm{MLC}_{20}\right)$ in buffer contained purified myosin and myosin light chain kinase (MLCK). (A) Effects of hesperidin on $\mathrm{p}-\mathrm{MLC}_{20}$ in different concentrations with incubation time (15 min). (B) Effects of 40 $\mu \mathrm{mol} / \mathrm{L}$ hesperidin on the $\mathrm{p}-\mathrm{MLC}_{20}$ in different incubation time points. (C) Effects of hesperidin on $\mathrm{Mg}^{2+}-$ ATP activity in different concentrations and incubation time points. Myosin $(5 \mu \mathrm{mol} / \mathrm{L})$ and myosin light chain kinase (MLCK, $2 \mu \mathrm{mol} / \mathrm{L})$ were used in these assays. Data in normal control group (no hesperidin treated) are set to a relative value of $100 \%$ (Normal control, NC) and expressed as the mean \pm SD. ${ }^{* * *} P<0.01$ compared with the NC. $n=4$ experiments. 
A

Without ML-7

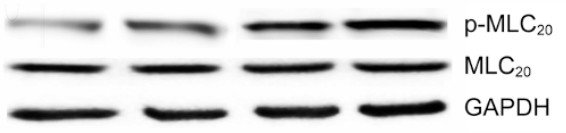

With ML-7
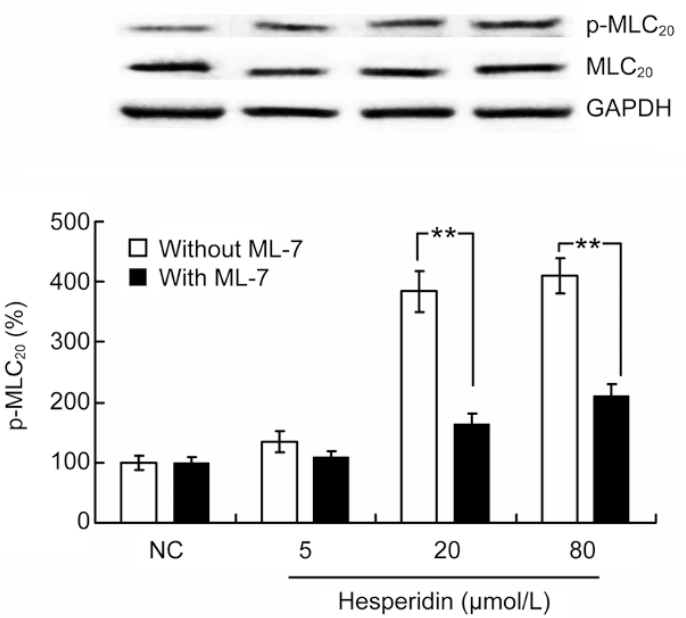

B si-Control

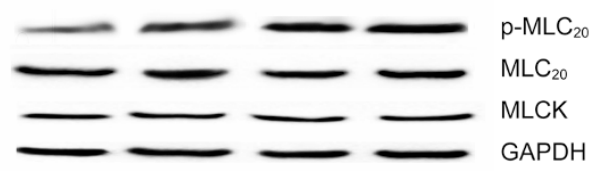

si-MLCK
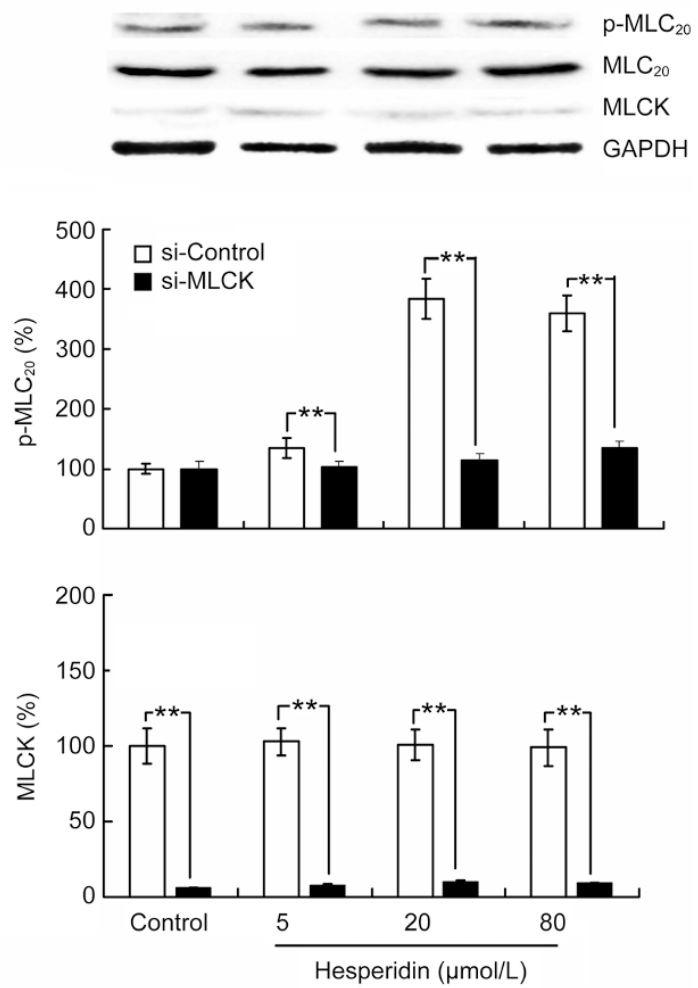

Figure 6. The role of MLCK in hesperidin induced contraction in fresh isolated intestinal smooth muscle cells. (A) Effects of hesperidin on the phosphorylation of 20-kDa regulatory light chain of myosin $\left(\mathrm{p}-\mathrm{MLC}_{20}\right)$ in the presence of myosin light chain kinase (MLCK) inhibitor ML-7. (B) Effects of hesperidin on the $\mathrm{p}-\mathrm{MLC}_{20}$ with siRNA knock down of MLCK. Data in control group (no hesperidin treated) are set to a relative value of $100 \%$ and expressed as the mean \pm SD. ${ }^{* *} P<0.01$ compared with the control. $n=4$ experiments.

nal surgery with manipulation of viscera postoperatively induces a state of digestive motor inactivity that may delay gastrointestinal transit ${ }^{[1]}$. The inflammatory phase of POI is initiated by mediators released from damaged tissue or by luminal bacterial products crossing an impaired gastrointestinal epithelium ${ }^{[2]}$. These damage signals activate inducible NO synthase and cyclooxygenase-2 pathways in macrophages ${ }^{[7]}$. Activated macrophages release NO and PGE-2, which directly inhibit neuromuscular function, finally leading to the bowel motility disorder ${ }^{[7,8]}$. Our results showed that the levels of the pro-inflammatory cytokines NO and prostaglandin PGE-2 in the POI rat cecum were significantly increased. However, there was no significant changes of these in the serum (data not shown). The casual relationship between inflammation and motility disorder in POI needs to be clarified. It was previously reported that the accumulation of pro-inflammatory cytokines and increased expression of $\mathrm{NO}$ could be reduced by hesperidin through inhibition of NO related pathways, which was also confirmed in our study ${ }^{[3-6]}$. We believe that hesperidin exerts anti-inflammatory effects on POI, ameliorating the digestive motor inactivity in POI rats and accelerating gastrointestinal transit. Restoration of the impaired bowel motility contributes to decreases in inflammatory responses. The reduction of iNOS, inflammatory cytokines and COX-2 may be just a marker for the decrease. The levels of pro-inflammatory cytokines, NO, and prostaglandins PGE-2 in the POI rat cecum could be significantly decreased by gavage administration of hesperidin, which suggests that hesperidin decreases the bowel inflammation in POI rats and that the effects are exerted partially by reducing the expression of iNOS and COX-2. However, the detailed mechanisms need further study.

POI is caused by an interplay of multiple factors, including neurogenic mechanisms, inhibition of GI motility, and intestinal inflammatory responses to bowel manipulation and surgical trauma. Accordingly, therapies that have been targeted to a single signaling molecule have shown limited promise. 
A
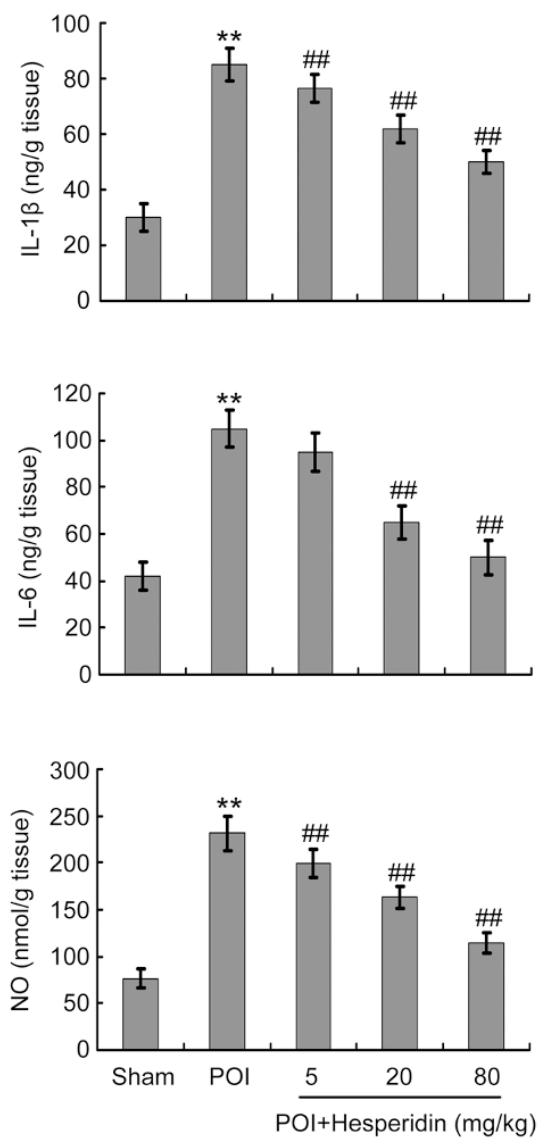

B
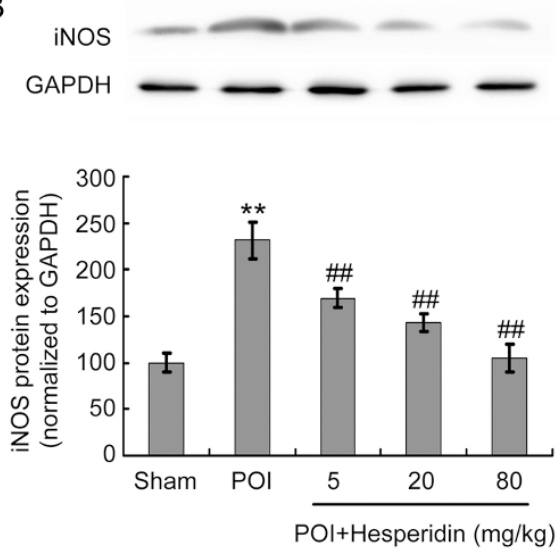
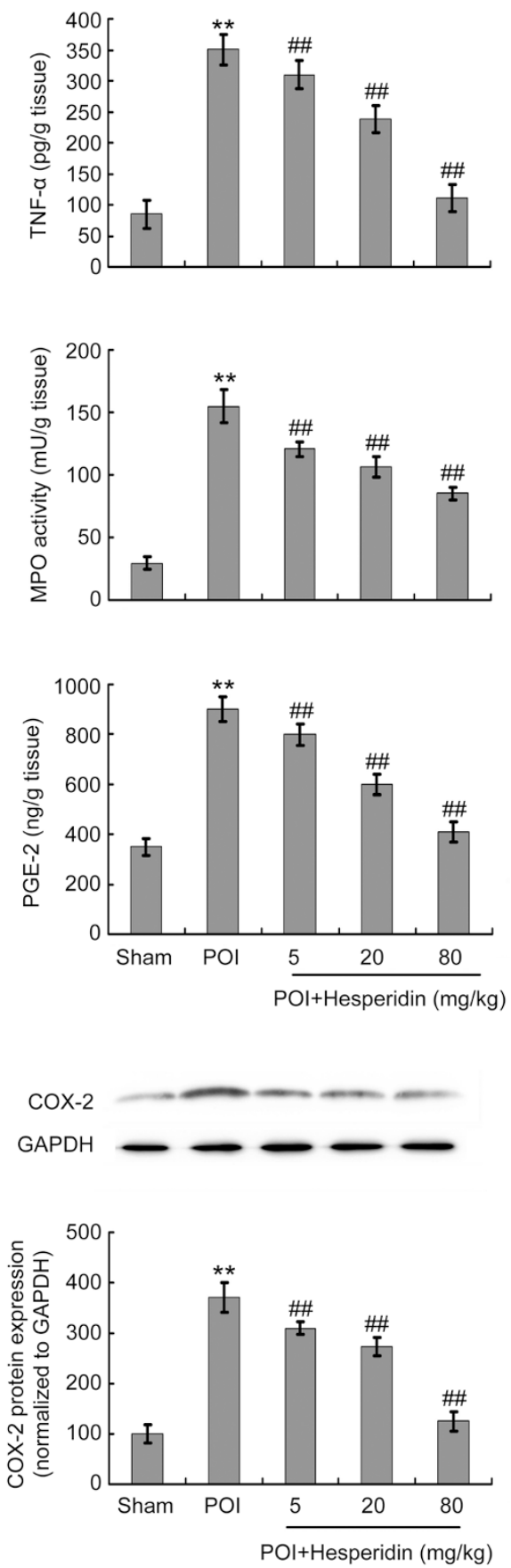

Figure 7. Effects of hesperidin on inflammatory responses in cecum tissue isolated from postoperative ileus (POI) rats. (A) Effects of hesperidin on the levels of pro-inflammatory cytokines, myeloperoxidase (MPO) activity and inflammatory mediators. (B) Effects of hesperidin on the expressions of iNOS and COX-2 in cecum isolated from POI rats. Data in sham-operated rats are set to a relative value of $100 \%$ and expressed as the mean \pm SD. ${ }^{* *} P<0.01$ compared with the sham; ${ }^{\# \#} P<0.01$ compared with POI group. $n=6$ rats.

Information obtained from the animal model in this study suggests that hesperidin, a natural product that shows no signs of toxicity with normal intake $\mathrm{e}^{[7,38]}$, may play an important role in multi-targeted POI treatment.

\section{Acknowledgements}

This study was supported by the National Natural Science
Foundation of China (grant number 31272392).

\section{Author contribution}

Da-peng CHEN and Jing-yu WANG designed the research; Yong-jian XIONG, Hong-wei CHU, Fang HAN, and Ya-chan LI performed research; Jing-yu WANG contributed reagents or analytical tools; Hong-wei CHU, Yuan LIN, Fu-jin WANG, 

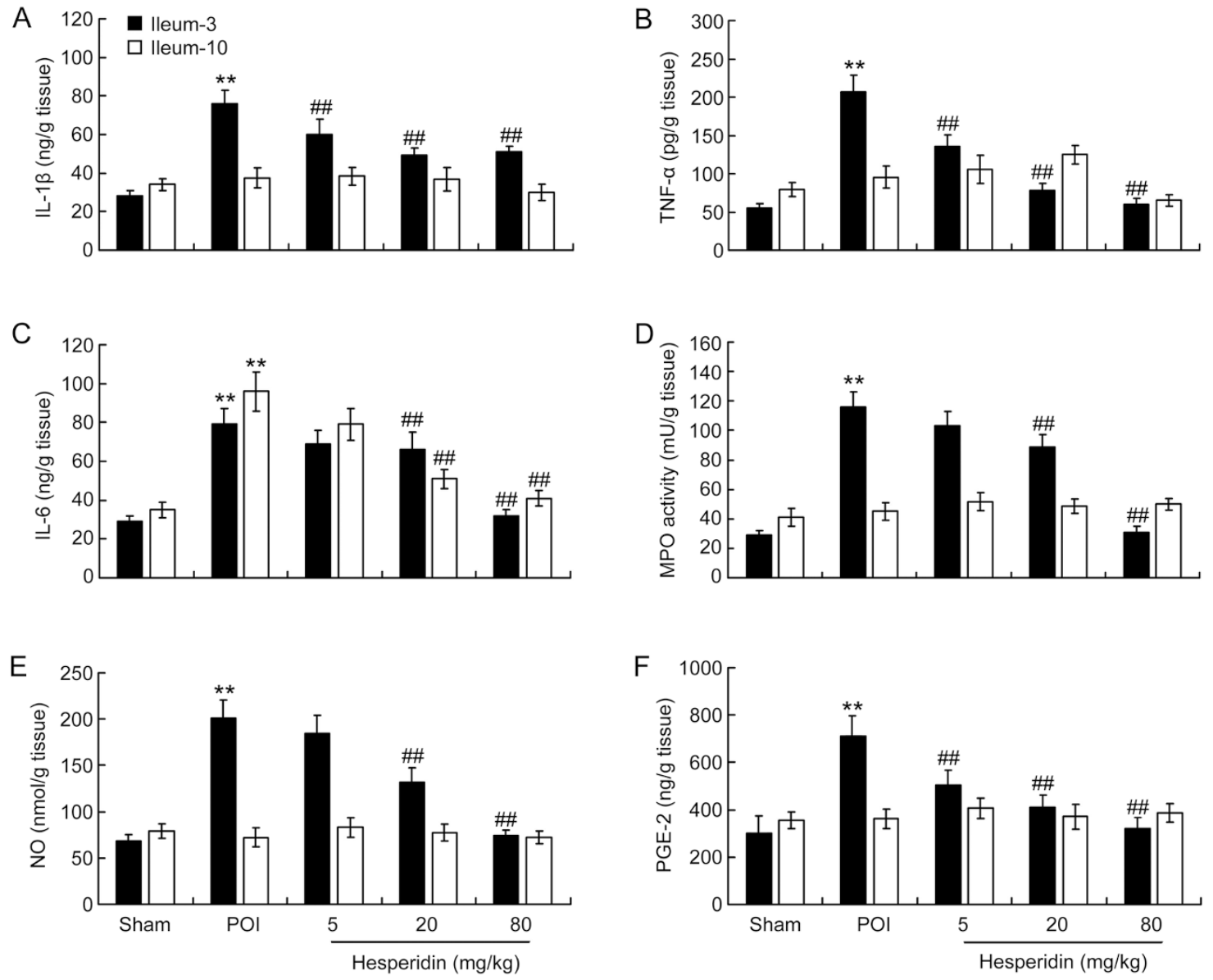

Figure 8. Effects of hesperidin on inflammatory responses in ileum tissue isolated from postoperative ileus (POI) rats. Data in sham-operated rats are set to a relative value of $100 \%$ and expressed as the mean \pm SD. Other data are the relative values compared with sham. ${ }^{* *} P<0.01$ compared with the sham; ${ }^{\# \#} P<0.01$ compared with POI group. $n=6$ rats. Ileum-3, ileum segment which was cut $3 \mathrm{~cm}$ proximal to the cecum; lleum-10, ileum segment which was cut $10 \mathrm{~cm}$ proximal to the cecum.

and Ai-guo WANG analyzed the data; and Da-peng CHEN and Yong-jian XIONG wrote the paper.

\section{References}

1 Kalff JC, Schraut WH, Billiar TR, Simmons RL, Bauer AJ. Role of inducible nitric oxide synthase in postoperative intestinal smooth muscle dysfunction in rodents. Gastroenterology 2000; 118: 316-27.

2 Miedema BW, Johnson JO. Methods for decreasing postoperative gut dysmotility. Lancet Oncol 2003; 4: 365-72.

3 Hilton WM, Lotan Y, Parekh DJ, Basler JW, Svatek RS. Alvimopan for prevention of postoperative paralytic ileus in radical cystectomy patients: a cost-effectiveness analysis. BJU Int 2013; 111: 1054-60.

4 Stengel A, Tache Y. Brain peptides and the modulation of postoperative gastric ileus. Curr Opin Pharmacol 2014; 19: 31-7.

5 Zeinali F, Stulberg JJ, Delaney CP. Pharmacological management of postoperative ileus. Can J Surg 2009; 52: 153-7.

6 Rychter J, Clave P. Intestinal inflammation in postoperative ileus: pathogenesis and therapeutic targets. Gut 2013; 62: 1534-5.

7 Garg A, Garg S, Zaneveld LJ, Singla AK. Chemistry and pharmacology of the Citrus bioflavonoid hesperidin. Phytother Res 2001; 15: 65569.

8 Orallo F, Alvarez E, Basaran H, Lugnier C. Comparative study of the vasorelaxant activity, superoxide-scavenging ability and cyclic nucleotide phosphodiesterase-inhibitory effects of hesperetin and hesperidin. Naunyn Schmiedebergs Arch Pharmacol 2004; 370: 452-63.

9 Bock C, Waldmann KH, Ternes W. Mangiferin and hesperidin metabolites are absorbed from the gastrointestinal tract of pigs after oral ingestion of a Cyclopia genistoides (honeybush tea) extract. Nutr Res 2008; 28: 879-91.

10 Zhang M, Zhang JP, Ji HT, Wang JS, Qian DH. Effect of six flavonoids on proliferation of hepatic stellate cells in vitro. Acta Pharmacol Sin 2000; 21: 253-6.

11 Zhao H, Zhang X, Chen X, Li Y, Ke Z, Tang T, et al. Isoliquiritigenin, a flavonoid from licorice, blocks M2 macrophage polarization in colitisassociated tumorigenesis through downregulating PGE2 and IL-6. Toxicol Appl Pharmacol 2014; 279: 311-21.

12 Middleton E Jr, Kandaswami C, Theoharides TC. The effects of plant flavonoids on mammalian cells: implications for inflammation, heart disease, and cancer. Pharmacol Rev 2000; 52: 673-751.

13 Oguzturk H, Ciftci O, Cetin A, Kaya K, Disli OM, Turtay MG, et al. Beneficial effects of hesperidin following cis-diamminedichloroplatinum-induced damage in heart of rats. Niger J Clin Pract 2016; 19: 99-103.

14 Bayomy NA, Elshafhey SH, Elbakary RH, Abdelaziz EZ. Protective effect of hesperidin against lung injury induced by intestinal ischemia/ 
reperfusion in adult albino rats: Histological, immunohistochemical and biochemical study. Tissue Cell 2014; 46: 304-10.

15 Hiroshige C, Hyounju K, Akiyo M, Satoko A, Yoshiko I, Kazuharu S, et al. Hesperidin prevents androgen deficiency-induced bone loss in male mice. Phytother Res 2014; 28: 289-95.

16 Donato F, Gomes MGD, Goes ATR, Filho CB, Fabbro LD, Antunes MS, et al. Hesperidin exerts antidepressant-like effects in acute and chronic treatments in mice: Possible role of $L$-arginine-NO-cGMP pathway and BDNF levels. Brain Res Bull 2014; 104: 19-26.

17 Subramanian P, Anandan R, Jayapalan JJ, Hashim OH. Hesperidin protects gentamicin-induced nephrotoxicity via Nrf2/HO-1 signaling and inhibits inflammation mediated by NF-KB in rats. J Funct Foods 2015; 13: 89-99.

18 Costa M, Furness JB. The peristaltic reflex: an analysis of the nerve pathways and their pharmacology. Naunyn Schmiedebergs Arch Pharmacol 1976; 294: 47-60.

19 Costa M, Furness JB. The sites of action of 5-hydroxytryptamine in nerve-muscle preparations from the guinea-pig small intestine and colon. Br J Pharmacol 1979; 65: 237-48.

20 Schemann M. Control of gastrointestinal motility by the "gut brain"-the enteric nervous system. J Pediatr Gastroenterol Nutr 2005; 41: S4-6.

21 Kitazawa T, Eto M, Woodsome TP, Khalequzzaman M. Phosphorylation of the myosin phosphatase targeting subunit and CPI-17 during $\mathrm{Ca}^{2+}$ sensitization in rabbit smooth muscle. J Physiol 2003; 546: 879-89.

22 Hansen MB. Neurohumoral control of gastrointestinal motility. Physiol Res 2003; 52: 1-30.

23 Plourde V, Wong HC, Walsh JH, Raybould HE, Tache Y. CGRP antagonists and capsaicin on celiac ganglia partly prevent postoperative gastric ileus. Peptides 1993; 14: 1225-9.

24 Trudel L, Tomasetto C, Rio MC, Bouin M, Plourde V, Eberling P, et al. Ghrelin/motilin-related peptide is a potent prokinetic to reverse gastric postoperative ileus in rat. Am J Physiol Gastrointest Liver Physiol 2002; 282: G948-52.

25 Umer A, Lugowska H, Sein-Anand J, Rekowski P, Ruczynski J, Petrusewicz J, et al. The contractile effects of several substituted short analogues of porcine galanin in isolated rat jejunal and colonic smooth muscle strips. Pharmacol Res 2005; 52: 283-9.

26 Xu K, Chang CM, Gao H, Shu HK. Epidermal growth factor-dependent cyclooxygenase-2 induction in gliomas requires protein kinase C-delta. Oncogene 2009; 28: 1410-20.

27 Wrobel MH, Grzeszczyk M, Mlynarczuk J, Kotwica J. The adverse effects of aldrin and dieldrin on both myometrial contractions and the secretory functions of bovine ovaries and uterus in vitro. Toxicol Appl Pharmacol 2015; 285: 23-31.

28 Ebashi S. A simple method of preparing actin-free myosin from smooth muscle. J Biochem 1976; 79: 229-31.

29 Yang JX, Wang XM, Tang ZY, Chen H, Xu H, Lin Y. The characterization of $\mathrm{Ca}^{2+}$-calmodulin independent phosphorylation of myosin light chains by a fragment from myosin light chain kinase. Sheng Wu Hua Xue Yu Sheng Wu Wu Li Xue Bao (Shanghai) 2003; 35: 793-800.

30 Tang ZY, Liu ZN, Fu L, Chen DP, Ai QD, Lin Y. Effect of lithium on smooth muscle contraction and phosphorylation of myosin light chain by MLCK. Physiol Res 2010; 59: 919-26.

31 Kobayashi K, Murata T, Hori M, Ozaki H. Prostaglandin E2-prostanoid EP3 signal induces vascular contraction via $\mathrm{nPKC}$ and ROCK activation in rat mesenteric artery. Eur J Pharmacol 2011; 660: 375-80.

32 Benham CD, Bolton TB, Lang RJ, Takewaki T. Calcium-activated potassium channels in single smooth muscle cells of rabbit jejunum and guinea-pig mesenteric artery. J Physiol 1986; 371: 45-67.

33 Ma T, Qi QH, Yang WX, Xu J, Dong ZL. Contractile effects and intracellular $\mathrm{Ca}^{2+}$ signalling induced by emodin in circular smooth muscle cells of rat colon. World J Gastroenterol 2003; 9: 1804-7.

34 Merritt JE, McCarthy SA, Davies MP, Moores KE. Use of Fluo-3 to measure cytosolic $\mathrm{Ca}^{2+}$ in platelets and neutrophils. Loading cells with the dye, calibration of traces, measurements in the presence of plasma, and buffering of cytosolic $\mathrm{Ca}^{2+}$. Biochem J 1990; 269: 513-9.

35 Cho YA, Choi DH, Choi JS. Effect of hesperidin on the oral pharmacokinetics of diltiazem and its main metabolite, desacetyldiltiazem, in rats. J Pharm Pharmacol 2009; 61: 825-9.

36 Parhiz H, Roohbakhsh A, Soltani F, Rezaee R, Iranshahi M. Antioxidant and anti-inflammatory properties of the citrus flavonoids hesperidin and hesperetin: an updated review of their molecular mechanisms and experimental models. Phytother Res 2015; 29: 323-31.

37 He WQ, Peng YJ, Zhang WC, Lv N, Tang J, Chen C, et al. Myosin light chain kinase is central to smooth muscle contraction and required for gastrointestinal motility in mice. Gastroenterology 2008; 135 : 610-20.

38 Morand C, Dubray C, Milenkovic D, Lioger D, Martin JF, Scalbert A, et al. Hesperidin contributes to the vascular protective effects of orange juice: a randomized crossover study in healthy volunteers. Am J Clin Nutr 2011; 93: 73-80. 